Relations industrielles

Industrial Relations

\title{
L'année sociale 1967, par Guy Spitaels et Simone Lambert, Études d'économie sociale, Éditions de l'Institut de Sociologie, Université Libre de Bruxelles, 1968, 350 pages.
}

\section{Bernard Solasse}

\section{Volume 23, numéro 4, 1968}

URI : https://id.erudit.org/iderudit/027977ar

DOI : https://doi.org/10.7202/027977ar

Aller au sommaire du numéro

\section{Éditeur(s)}

Département des relations industrielles de l'Université Laval

ISSN

0034-379X (imprimé)

1703-8138 (numérique)

Découvrir la revue

Citer ce compte rendu

Solasse, B. (1968). Compte rendu de [L'année sociale 1967, par Guy Spitaels et Simone Lambert, Études d'économie sociale, Éditions de l'Institut de Sociologie, Université Libre de Bruxelles, 1968, 350 pages.] Relations industrielles /

Industrial Relations, 23(4), 708-708. https://doi.org/10.7202/027977ar

Tous droits réservés @ C Département des relations industrielles de l'Universite Laval, 1968
Ce document est protégé par la loi sur le droit d'auteur. L'utilisation des services d'Érudit (y compris la reproduction) est assujettie à sa politique d'utilisation que vous pouvez consulter en ligne.

https://apropos.erudit.org/fr/usagers/politique-dutilisation/ 
de la satisfaction au travail, de la motivation et de l'exercice de l'autorité. On termine cette partie en traitant des problèmes de communications, de discipline et de la façon de réduire la résistance oux changements.

Les parties IV, $V$ et $V I$ traitent plus spécifiquement des problèmes de l'entreprise et des méthodes modernes qui ont été dévelup. dées pour permettre de les résoudre. On y traite surtout du rôle de l'administration du personnel et des diverses activités qu'elle comporte: recrutement, entraînement, formation, appréciation du personnel administration des solaires, techniques d'incitation à un rendement supérieur, etc.

Lo septième partie constitue en quelque sorte la conclusion du volume et traite des responsabilités de la gérance.

\section{André PETIT}

L'année sociale 1967, par Guy Spitaels et Simone Lambert, Etudes d'économie sociole, Editions de I'Institut de Sociologie, Université Libre de Bruxelles, 1968, 350 pages.

Cet ouvrage est le huitième d'une série qui débuta en 1960. II comprend cinq parties intitulées réciproquement: La vie législative, la vie paritaire, la vie syndicale et les programmes sociaux, le visage social de l'Europe, l'emploi et le chômage.

Les outeurs n'entendent nullement entreprendre une étude systématique des événements et des législations qu'ils analysent: leur démarche est essentiellement descrip- tive et chronologique. L'ensemble, semble$t$-il objectif, constitue un instrument de travail précieux pour le chercheur ou pour quiconque se préoccupe de la vie sociale et syndicale en Belgique. Son intérêt pourrait être sensiblement accrû en incluant en onnexe ou à la fin de chaque étude porticulière des références bibliographiques plus précises.

\section{Bernard SOLASSE}

The Unions, Structure, Development, and Management, by Marten Estey, Hartcourt, Broce \& World Inc., New York, 1967, 125 pages.

Cet ouvrage a d'abord été conçu pour aider les étudiants. Après une brève introduction au mouvement syndical américain, i) trace un tableau de l'évolution des modèles d'organisation. Ensuite, il fait l'anatomie du syndicalisme et explique comment les syndicats sont administrés. Deux chapitres sont consacrés au comportement des syndicats ainsi qu'à leur rôle vis-à-vis de I'Etat. L'ouvrage se termine avec quelques pages sur l'avenir du syndicalisme.

L'auteur connaît bien le syndicalisme américain. Son ouvrage est bien divisé et très schématique. II ne fait pas avancer les connaissances en ce domaine, mais il réfère oux bons auteurs sur le sujet. II peut être utile à ceux qui désirent s'initier au syndicalisme. On remarquera cependant qu'il se limite strictement au syndicalisme des EtatsUnis sans même faire des comparaisons avec ce qui existe ailleurs.

\section{Gérard DION}

\section{PUBLICATIONS RECENTES}

\section{GENERALITES}

\& Technological Change and Learning in the Computer Industry », by James L. Barr $\xi$ Kenneth E. Knight, Management Science, vol. 14, no 11, July 1968, pp. 661-682.

- Flexible Pricing of Computer Services $\gg$, by Seymour Smidt, Management Science, vol. 14, no 10, June 1968, pp. B-581-B-600.

- Technological Change and the Level of Employment in Western Europe $\gg$, by Felix
Paukert, British Journal of Industrial Relations, vol. VI, no 2, July 1968, pp. 139-156.

«The Swedish Experience with Industrial Democracy 》, by Richard B. Peterson, The British Journal of Industrial Relations, vol. VI, no 2, July 1968, pp. 185-204.

«Technical Progress and Technical Schools», by Svetozar Pejovich, Review of Social Economy, vol. XXVI, no 1, March 1968, pp. 4050. 